\title{
Deciphering the role of endogenous opioids in high frequency TENS using low and high doses of naloxone
}

\author{
Guillaume Leonard ${ }^{1}$, Philippe Goffaux ${ }^{1}$, Serge Marchand ${ }^{1}$
}

${ }^{1}$ Université de Sherbrooke, Faculté de médecine, Sherbrooke, Quebec, Canada, J1H 5N4.

Keywords: Transcutaneous electrical nerve stimulation (TENS); naloxone; endogenous opioids; opioid receptors

Pages: 22

Tables: 1

Figures: 3

Corresponding author: $\quad$ Serge Marchand, Ph.D.

Université de Sherbrooke

Faculté de médecine, neurochirurgie

3001, 12e avenue nord

Sherbrooke (Québec) Canada J1H 5N4 


\section{Introduction}

Transcutaneous electrical nerve stimulation (TENS) is a non-invasive modality commonly used in rehabilitation for pain relief [20]. Scientific rationale behind lowintensity/ high-frequency TENS (also known as conventional-mode TENS) is generally based on the gate-theory proposed by Melzack and Wall [19] where stimulation of nonnociceptive afferents (A $\beta$ fibers) inhibit the nociceptive signal transmitted by $\mathrm{A} \delta$ and $\mathrm{C}$ fibers. The release of endogenous opioids following high-frequency TENS application has also been proposed as a possible mechanism of action [9;22]. This latter premise was, however, refuted by the work of several authors who showed that the analgesic properties of high-frequency TENS could not be reversed by the opioid antagonist naloxone $[1 ; 10 ; 13 ; 14 ; 17 ; 24 ; 29]$.

Recently, Sluka and colleagues $[15 ; 25]$ have re-examined the role of endogenous opioids in the analgesia induced by high-frequency TENS. Using a kaolin and carrageenan joint inflammation model in Sprague-Dawley rats, they showed that spinal [25] and supraspinal [15] blockade of $\delta$ opioid receptors with naltrindole reversed highfrequency TENS analgesia. They also reported that high doses of naloxone (blocking the $\mu, \delta$ and $\kappa$ opioid receptors) prevented the antihyperalgesic effects produced by highfrequency TENS whereas lower doses of naloxone (blocking only the $\mu$ opioid receptors) did not reversed the effect of high-frequency TENS [25]. These results lead to the conclusion that the analgesia induced by high-frequency TENS was mediated by $\delta$ receptors and that failure of naloxone to reverse high-frequency TENS analgesia in previous studies was due to the inability of the antagonist to block $\delta$ receptors with the low doses used. 
Based on these results from animal studies, the purpose of this study was to reinvestigate the implication of opioid receptors in the analgesia induced by highfrequency TENS in human subjects by using high $(0.14 \mathrm{mg} / \mathrm{kg})$ and low $(0.02 \mathrm{mg} / \mathrm{kg})$ doses of naloxone. Considering the work of Sluka and colleagues $[15 ; 25]$ and based on previous naloxone studies in humans $[1 ; 10 ; 13 ; 14 ; 17 ; 24 ; 29]$, we hypothesized that the high - but not the low - dose of naloxone would reverse the analgesia induced by highfrequency TENS.

\section{Methods}

\section{Participants}

Twenty-one healthy volunteers (mean age $25.2 \pm 6.0$ years; 12 males) participated in the study. None of the participants suffered from any known diseases and none took medications. The experiment took place at the Clinical Research Centre of the Sherbrooke University Hospital, Sherbrooke, Quebec, Canada. Subjects were recruited through local ads and were all French speaking community-dwelling individuals. The local Institutional Ethics Committee approved the study's procedures and each participant provided informed consent before participation.

\section{Tonic heat pain model}

Participants were seated comfortably in a reclining chair. Before each session, a pre-testing session was first provided in order to familiarize participants with the computerized visual analogue scale (COVAS; Medoc, Advanced Medical Systems, Minneapolis) and to determine the temperature which would be used during the two 
minutes pain test. This pre-test was performed with a $1 \mathrm{~cm}^{2}$ Peltier-type thermode (Medoc, Advanced Medical Systems, Minneapolis) applied on the lateral surface of the left forefoot. Participants were advised that the thermode temperature would gradually increase from $32^{\circ} \mathrm{C}$ to $51^{\circ} \mathrm{C}$ (rising rate $=0.3^{\circ} \mathrm{C} / \mathrm{s}$ ). The pre-test was repeated twice during which the subjects reported verbally their pain threshold and pain tolerance threshold. On the third pre-test, subjects were given the COVAS and advised that they would have to start moving the cursor towards to right (towards the "100" mark) when they started to feel pain (pain threshold) and that the cursor had to be at the extreme right (at the "100" mark) when pain was intolerable (pain tolerance). This procedure was repeated until the subject's pain reports were consistent between trials. The temperature used during the following experimental heat pain test was determined by selecting the temperature for which the subject had rated the pain intensity at 50/100 with the COVAS (see Tousignant-Laflamme et al. [27] for a similar approach).

Following the pre-test, participants were given a 10 minute rest period before the experimental test began. The tonic pain test was then performed with the application of the thermode at constant temperature applied on the lateral aspect of the left heel for 2 min. Subjects were told that the thermode temperature could rise, remain stable or decrease and that they would have to evaluate their pain with the COVAS throughout the 2 min test. In fact, after a constant rise $\left(0.3^{\circ} \mathrm{C} / \mathrm{s}\right)$ from baseline $\left(32^{\circ} \mathrm{C}\right)$ to the individually predetermined temperature, the thermode's temperature remained constant throughout the 120 seconds. 


\section{TENS stimulation protocol}

For each visit, TENS stimulations were delivered using a pair of electrodes (rubber silicone, $4 \times 4 \mathrm{~cm}$ ) connected to a digital Eclipse Plus apparatus (Empi, St-Paul, Minnesota). Electrodes were placed over the lateral aspect of the foot, above and below the area to be stimulated by the thermode. More specifically, the cathode was positioned proximally near and above the malleolus and the anode was placed distally near the fifth metatarsal's tuberosity (see Figure 1). With the frequency set at $100 \mathrm{~Hz}$ and the pulse duration at $60 \mu \mathrm{s}$, the stimulator's intensity was adjusted to produce a strong and comfortable tingling sensation in the territory innervated by the sural nerve. The experimenter made sure that the paresthesias were non-painful and covered adequately the lateral aspect of the heel. In cases where stimulation did not cover properly the region, the stimulator was turned OFF and the electrodes repositioned. The stimulation was applied for 25 minutes and the intensity was occasionally raised (based on the participant's sensation) to account for nerve accommodation and to maintain the same level of paresthesias.

\section{Experimental design}

Volunteers were randomly assigned in a triple-blind, cross-over design to one of the following conditions: (1) high dose of naloxone $(0.14 \mathrm{mg} / \mathrm{kg})$, (2) low dose of naloxone $(0.02 \mathrm{mg} / \mathrm{kg})$ and (3) saline $(0.9 \% \mathrm{NaCl})$. Randomization (controlling for

presentation order) was made using Minitab Statistical Sotfware (version 15.0 for Windows ${ }^{\circledR}$, State College, PA, USA). Controlling for presentation order ensured that an equal number of participants were randomized to each possible permutation level. All 
injections had an equivalent volume and were administered by a registered nurse using an intravenous catheter inserted in the right forearm. The naloxone dosage $(0.14 \mathrm{mg} / \mathrm{kg})$ was chosen because it has been used successfully in previous studies to completely antagonize endogenous opioid-mediated analgesia in healthy volunteers [2;4] and because it is believed to ensure adequate blockage of all opioid receptor subtypes [3;5]. Because of naloxone's short half-life (c. 30-40 min), all injections (including saline) were administered twice during the session (approx. 35 minutes interval). This ensured that naloxone maintained its full effect throughout the testing session. The experimenter, nurse and participant were blinded to the substance being injected. Every participant was submitted to the three conditions (high dose of naloxone, low dose of naloxone, saline) in three consecutive sessions (approx. 120 minutes, 1 week interval between each visit). Each time, the tonic heat pain test was performed on four occasions: (1) at baseline (i.e. 10 minutes after the pre-testing session), (2) after injection (naloxone or saline), (3) during TENS (i.e. 5 minutes prior to the end of TENS stimulation) and (4) 10 minutes after TENS application.

\section{Data analysis}

To facilitate comparisons, pain intensity ratings obtained during the 2 minute stimulation test were averaged and the mean was used in subsequent analysis. Because of the relatively small number of subjects included in the study $(\mathrm{n}<30)$ and since visual inspection of the histograms did not allow us to assume that the data were normally distributed, non-parametric tests were employed. Differences were considered to be 
significant if $\mathrm{p}<0.05$ was obtained. All tests were performed using SPSS (version 13.0 for Windows ${ }^{\circledR}$, Chicago, IL, USA).

\section{Results}

Conditioning effect

Between-subject analyses were first performed to determine if the order of presentation affected the pattern of results obtained. To do this, delta pain scores representing pain reductions experienced during TENS stimulation for the saline condition (delta pain score for saline $=$ pain at baseline - pain during TENS) were calculated and compared between (i) participants who received saline at their first session, (ii) participants who received saline at their second session (after having received a high or a low dose of naloxone at their first session) and (iii) participants who received saline at their third session (after having received naloxone at their first and second session).

Figure 2 illustrates the analgesic effect of TENS obtained during stimulation for the saline condition between the three groups. As it can be seen from the figure, the large analgesic effect of TENS observed in participants who received saline at their first visit was almost completely absent in participants who received saline after having received naloxone. These differences were confirmed by the Kruskall-Wallis Test $(p<.05)$ and by the post-hoc Mann-Whitney test which revealed a significant difference between participants who received saline at their first session and participants who received naloxone at their first and second sessions and saline at their third session $(p<.01)$. 


\section{Effect of naloxone on TENS analgesia}

Since the order of presentation greatly affected the pattern of results and because we did not want our results to be contaminated by carry-over effects, we analysed the first testing session only. To compensate for the lower power of the new analyses compared with the intended cross-over design analyses, three other participants were recruited (new sample $=24$ healthy volunteers, mean age $24.9 \pm 5.7$ years; 13 males). These three participants were submitted to the same experimental protocol described above except that they took part in only one testing session (i.e. the first testing session).

The pain intensity ratings obtained at baseline showed that every participant experienced pain (all pain measures at baseline $\geq 31$ ). Thermode temperatures and TENS stimulation intensities for all three conditions (at session 1 only) are showed in Table 1. The Kruskall-Wallis Tests revealed that there were no significant differences in any of these parameters across the three groups (all p-values $\geq .38$ ).

The average pain intensity obtained at baseline, post-injection, during TENS and post-TENS are presented in Figure 3. As it can be seen from this figure, the high dose of naloxone - but not the low dose - blocked the analgesic effect of TENS seen during the saline condition. Our Friedman's Tests confirmed that there was a large and significant change in pain intensity across the different time measures for the saline $\left(\mathrm{p}<.001, \eta^{2}=.90\right)$ and low dose group $\left(p<.05, \eta^{2}=.78\right)$ but not for the high dose group $\left(p=.20, \eta^{2}=.66\right)$. Post-hoc Wilcoxon Tests showed that there was a significant reduction in pain intensity both during and after TENS when compared with baseline for the saline (all p-values $=.01$ ) and low dose group (all p-values $<.05$ ). There was no difference between the pain 
measures obtained at baseline and the pain measures obtained 10 minutes after the injection (all p-values $>.23$ ).

Between-subject analyses across the three groups using Kruskall-Wallis Tests revealed significant differences for pain intensity during TENS $(\mathrm{p}<.05)$ but not for the other time measures (baseline, post-injection and post-TENS, all p-values >.63), suggesting that naloxone only had an effect when TENS was applied. Post-hoc MannWhitney Tests comparing pain intensity during TENS showed that there was a significant difference between the saline group and the high dose group $(\mathrm{p}<.01)$. There was no difference between the saline group and the low dose group $(\mathrm{p}=.08)$ and between the low dose group and high dose group $(\mathrm{p}=.12)$.

\section{Discussion}

In this study, we sought to determine if naloxone could reverse the analgesia induced by high-frequency TENS. Our results showed that the high - but not the low dose of naloxone blocked high-frequency TENS analgesia, hence providing the first evidence that the analgesia induced by high-frequency TENS in humans is mediated by opioid receptors.

For several years and until recently, high-frequency TENS was believed to be non-opioidergic. This supposition was based on numerous studies $[1 ; 10 ; 13 ; 14 ; 17 ; 24 ; 29]$ which revealed that the analgesic properties of high-frequency TENS could not be reversed by low doses of naloxone. On the opposite, other reports showed that the same doses of naloxone could block the analgesic effect of low-frequency/ high-intensity TENS (also-called acupuncture-like TENS) [7;17;23;29] leading to the premise that low- 
frequency TENS analgesia was produced via mechanisms utilizing endogenous opioids whereas high-frequency TENS analgesia was mediated via some other mechanism [24]. Recently, Sluka et al. [25] challenged this long-standing belief by showing that highfrequency TENS analgesia in Sprague-Dawley rats could be reversed by the opioid antagonist naloxone but that this required higher doses than what was necessary to reverse low-frequency TENS analgesia. They also showed that selective blockade of $\delta$ opioid receptors with naltrindole reversed the analgesia induced by high-frequency TENS. Sluka et al. interpreted these findings as an indication that high and lowfrequency TENS analgesia were mediated by different opioid receptors, with lowfrequency TENS producing analgesia through $\mu$ opioid receptors and high-frequency TENS producing analgesia through $\delta$ opioid receptors. Indeed, the affinity of naloxone for $\delta$ opioid receptors is lower than that for $\mu$ opioid receptors [11], and it is likely that the inability to reverse high-frequency TENS analgesia in previous human studies $[1 ; 10 ; 13 ; 14 ; 17 ; 24 ; 29]$ be attributable to the fact that the doses used were insufficient to antagonize the $\delta$ opioid receptors. Unfortunately, no human studies followed the work of Sluka and colleagues and it was impossible, until today, to confirm that high-frequency TENS analgesia was opioid-mediated in humans. Our results corroborate and extend the findings of Sluka et al. by showing that high-frequency TENS analgesia in humans can be reversed by high doses of naloxone. They are also coherent with the results of previous human studies which showed that low naloxone doses not reverse highfrequency TENS analgesia $[1 ; 10 ; 13 ; 14 ; 17 ; 24 ; 29]$. Manifestly, the insufficient amount of opioid antagonist prevented previous human studies from discovering the importance of opioid receptors in producing high-frequency TENS analgesia. 
These results have several important implications. First, they allow a better understanding of the mechanisms of action of TENS, providing the neurophysiological basis for the utilisation of TENS in clinic. They also open interesting therapeutic considerations. For instance, the involvement of opioid receptors in TENS analgesia suggests the existence of a possible interaction between TENS and oral opioid use. Animal evidence already support such a hypothesis, with studies showing (i) that animals rendered tolerant to high and low-frequency TENS are tolerant to $\delta$ and $\mu$ opioid agonists, respectively [6], and (ii) that rats tolerant to morphine are also tolerant to low-frequency TENS [26]. Future studies are necessary to determine if the interaction found in rodents between TENS and oral opioid use is also present in humans.

\section{Conditioning effect}

One important observation was that the analgesic effect of TENS stimulation (measured during the saline condition) was stronger when the saline was given during the first session than when it was given during the second or third session. Pre-exposition to ineffective TENS treatment (in this case because of prior opioid blockade with naloxone) would hence reduce the efficacy of subsequent stimulation, even if given several days later in the absence of opioid blockade. This phenomenon, which is probably due to a conditioning effect where exposition to unsuccessful procedures reduce the efficacy of subsequent ones, has already been observed in a study by Charron et al. [8] for placebo analgesia. In the present study, we show that this conditioning effect can not only decrease the efficacy of placebo treatments but also the efficacy of genuine interventions (i.e. TENS treatment). 
Although exploratory, these results have two important implications. First, they suggest that the analgesic effect of TENS was underestimated in our participants who previously received naloxone. This shortcoming brought us to discard the intended cross-over design analyses and to retain only the first visit of every participant. Because this conditioning effect is susceptible to occur in other research situations, researchers should be cautious when they choose to use cross-over designs. Moreover, and from a clinical perspective, these observations would entail that prior exposition to ineffective treatments (for example ineffective TENS applications because of inadequate electrode positioning or because of prior caffeine consumption [18]) would significantly reduce the analgesic efficacy of subsequent treatments. This kind of observation evidently reminds the clinician about the importance of maximizing the efficacy of the first treatment. Future research aimed specifically at evaluating the interaction between the analgesic effect of a treatment and the previous experience of the patient with this treatment is needed.

\section{Limitations}

On potential limitation of the present study is that TENS intensity was systematically raised during the stimulation period to maintain the same level of parasthesias. Even though this might seem problematic, increasing TENS intensity during stimulation is a common procedure performed in clinic to compensate for nerve accommodation [21;28]. Most importantly however, this procedure can not account for the pattern of results obtained. That is, we observed a reduction in TENS analgesia 
during the high dose condition despite the fact that we raised TENS intensity (a procedure which is thought to potentiate TENS analgesia [21]).

Perhaps another important criticism that could be addressed to the present study is the use of an experimental pain paradigm rather than a clinical pain paradigm. Indeed, several of the previous studies looking at the interaction between high-frequency TENS and naloxone employed clinical pain paradigms $[1 ; 10 ; 13 ; 14 ; 17 ; 24]$ and some could wonder if the results of this study (based on pain ratings obtained on healthy volunteers during prolonged heat stimulation) would also have been obtained in pain patients. Without refuting the fact that experimental pain paradigm have less external validity than clinical pain paradigms, one has to remember that the former has on the counterpart the advantage of increasing the internal validity due to the constant stimulation parameters employed at each time measure. In contrast, clinical pain conditions (even if fairly stable) can fluctuate throughout an experimental session, thus decreasing internal validity. Nevertheless, the decision to recruit healthy volunteers rather than clinical pain patient was above all based on ethical reasons. Indeed, high doses of naloxone have been shown to increase clinical pain in some patients $[12 ; 16]$.

One more limitation concerns the relatively low power of the analyses comparing pain scores between the low dose group and the other groups. In fact, power calculation revealed that comparison between the low dose and the saline group reached a power of $38 \%$ and that comparison between the low dose and high dose group reached a power of 41\%. Alternately, comparison between the high dose and saline reached appropriate statistical power $(1-\beta=89 \%)$. Although increasing the sample size may reveal significant effects when comparing the low dose condition to all other conditions, it 
should not affect the dose-response relationship identified here. We believe this doseresponse relationship results from fundamental inter-condition differences in effect size, not sample size. This is further confirmed by the index of effect size provided (see Results section). Evidence of a dose response relationship is also consistent with the idea that naloxone is a non-specific antagonistic which incrementally affects opioid receptors [11].

Finally, it should be pointed out that the present study does not allow us to identify which opioid receptor ( $\mu, \delta$ or $\kappa)$ mediated TENS analgesia. Even though the observations made on Sprague-Dawley rats by the group of Sluka and colleagues using the selective opioid antagonist naltrindole would suggest that the analgesia induced by high-frequency TENS in our group of participants was subserved by the $\delta$ opioid receptor [15;25], the impossibility to use selective antagonists in human subjects prevents us from making any clear assertion.

\section{Conclusion}

Our results showed that the high - but not the low - dose of naloxone blocked highfrequency TENS analgesia, indicating that the analgesic effect of this type of stimulation involves endogenous opioids. Manifestly, the insufficient amount of opioid antagonist used in the past prevented previous studies from discovering the importance of opioid receptors in producing high-frequency TENS analgesia. Finally, the important reduction in TENS analgesia in participants who received saline at their third session (after having received naloxone at their first and second session) suggest that previous exposition to ineffective treatments can substantially decrease the efficacy of subsequent interventions. 
This underscores the importance of previous experience on pain relief and reminds the clinician about the necessity of maximizing the efficacy of the first treatment.

\section{Acknowledgments}

The authors wish to thank Stéphanie Racette and Yannick Tousignant-Laflamme for their thoughtful comments on the manuscript and Marie-Ève Mauger for her help with data collection. Part of this work served as a partial fulfillment of Ph.D. degree by Guillaume Léonard. Guillaume Léonard received postgraduate scholarships from FRSQ (Fonds de recherche en santé du Québec) and IRSST (Institut de recherche en santé et sécurité au travail). Serge Marchand is supported by CIHR (Canada) and FRSQ (Québec) and is a member of the Centre de recherche clinique Étienne-Le-Bel (CRCELB) of the CHUS. We have no conflict of interest to report. 
Figures

Figure 1. TENS and thermode application

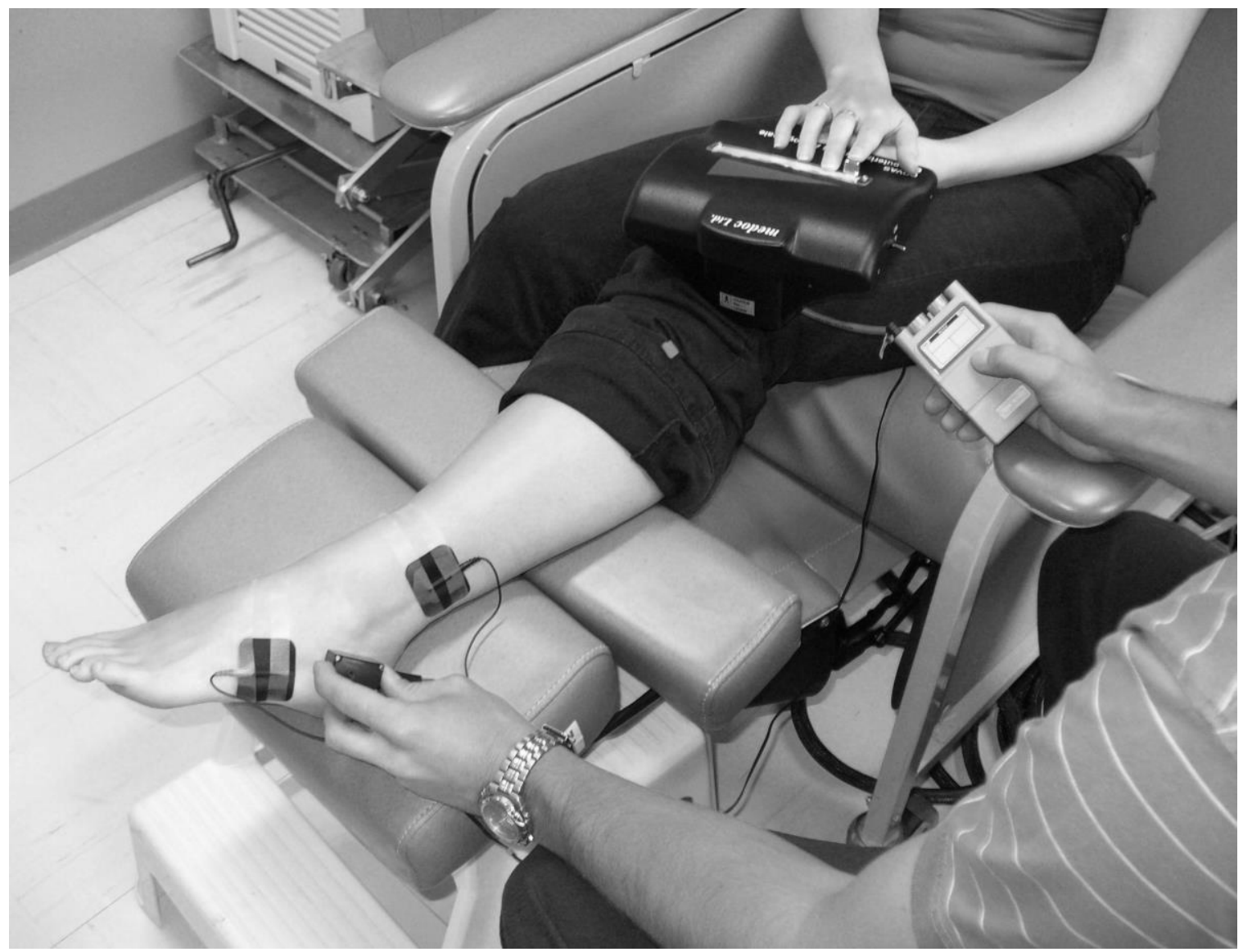


Figure 2. TENS analgesia experienced during the saline condition (control condition) between participants who received (1) saline at their first session, (2) naloxone at their first session and saline at their second session and (3) naloxone at their fist and second sessions and saline at their third session. Participants who received saline at their first session showed a larger analgesic effect than the other participants.

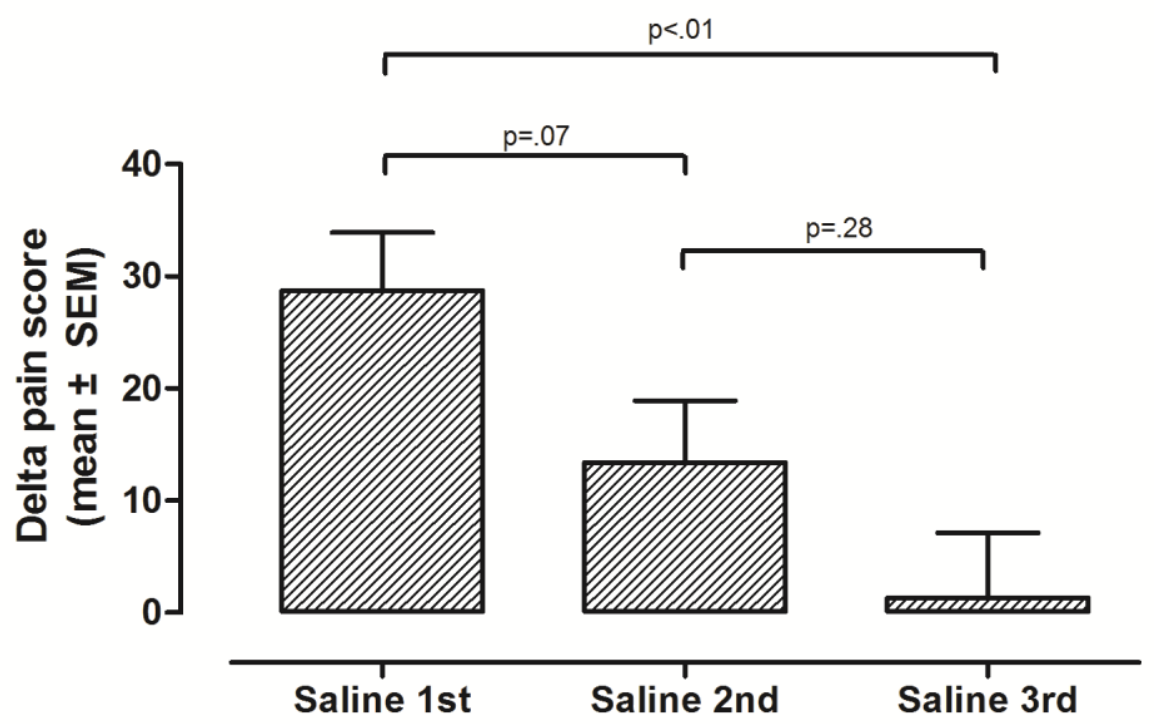


Figure 3. Comparisons of pain intensity measures to thermal stimulation obtained at baseline, after injection, during TENS and after TENS. There was a significant reduction in pain intensity both during and after TENS when compared with baseline for the saline $(\mathrm{p}<.01)$ and low dose condition $(\mathrm{p}<.05)$ but not for the high dose condition $(\mathrm{p}=.20)$. Between-subject analyses comparing pain intensity during TENS across the three groups revealed that there was a significant difference during TENS between the saline group and the high dose group $(\mathrm{p}<.01)$.

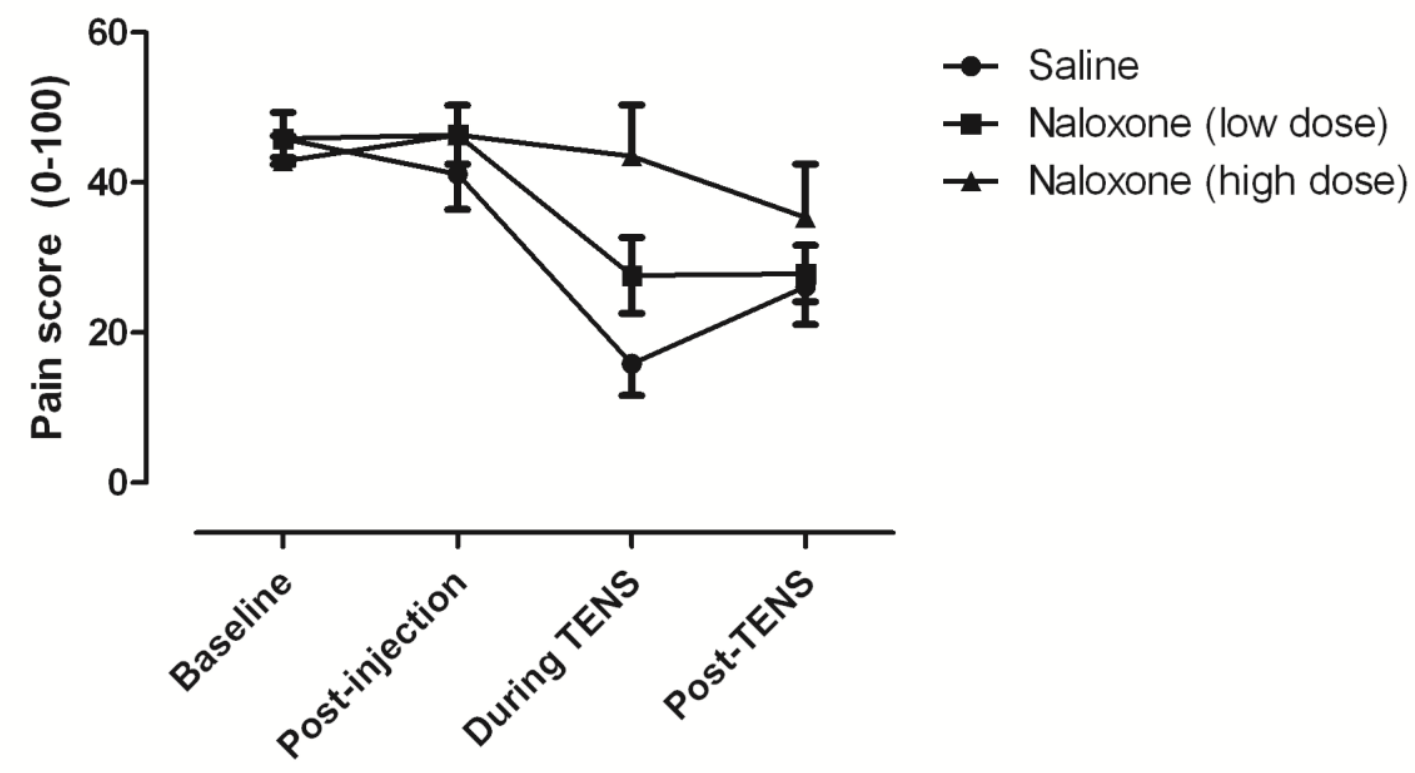




\section{References}

[1] Abram SE, Reynolds AC, Cusick JF. Failure of naloxone to reverse analgesia from transcutaneous electrical stimulation in patients with chronic pain. Anesth Analg 1981 Feb;60(2):81-4.

[2] Amanzio M, Pollo A, Maggi G, Benedetti F. Response variability to analgesics: a role for non-specific activation of endogenous opioids. Pain 2001 Feb 15;90(3):205-15.

[3] Benedetti $F$. The opposite effects of the opiate antagonist naloxone and the cholecystokinin antagonist proglumide on placebo analgesia. Pain 1996 Mar;64(3):535-43.

[4] Benedetti F, Arduino C, Amanzio M. Somatotopic activation of opioid systems by target-directed expectations of analgesia. J Neurosci 1999 May 1;19(9):3639-48.

[5] Bruehl S, Chung OY, Ward P, Johnson B, McCubbin JA. The relationship between resting blood pressure and acute pain sensitivity in healthy normotensives and chronic back pain sufferers: the effects of opioid blockade. Pain 2002 Nov;100(1-2):191-201.

[6] Chandran P, Sluka KA. Development of opioid tolerance with repeated transcutaneous electrical nerve stimulation administration. Pain 2003 Mar;102(12):195-201.

[7] Chapman CR, Benedetti C. Analgesia following transcutaneous electrical stimulation and its partial reversal by a narcotic antagonist. Life Sci 1977 Dec $1 ; 21(11): 1645-8$.

[8] Charron J, Rainville P, Marchand S. Direct comparison of placebo effects on clinical and experimental pain. Clin J Pain 2006 Feb;22(2):204-11.

[9] Facchinetti F, Sandrini G, Petraglia F, Alfonsi E, Nappi G, Genazzani AR. Concomitant increase in nociceptive flexion reflex threshold and plasma opioids following transcutaneous nerve stimulation. Pain 1984 Jul;19(3):295-303.

[10] Freeman TB, Campbell JN, Long DM. Naloxone does not affect pain relief induced by electrical stimulation in man. Pain 1983 Oct;17(2):189-95.

[11] Gourlay GK. Advances in opioid pharmacology. Support Care Cancer 2005 Mar;13(3):153-9.

[12] Gracely RH, Dubner R, Wolskee PJ, Deeter WR. Placebo and naloxone can alter post-surgical pain by separate mechanisms. Nature 1983 Nov 17;306(5940):2645. 
[13] Hansson P, Ekblom A, Thomsson M, Fjellner B. Influence of naloxone on relief of acute oro-facial pain by transcutaneous electrical nerve stimulation (TENS) or vibration. Pain 1986 Mar;24(3):323-9.

[14] Honig S, Zeale P, Mason A, Fitzgerald D, Millet I, Echemendia E. Highfrequency transcutaneous electrical nerve stimulation: Lack of correlation with serum beta-endorphin levels and failure of analgesia reversal with naloxone. Clinical Journal of Pain 1987;2:215-7.

[15] Kalra A, Urban MO, Sluka KA. Blockade of opioid receptors in rostral ventral medulla prevents antihyperalgesia produced by transcutaneous electrical nerve stimulation (TENS). J Pharmacol Exp Ther 2001 Jul;298(1):257-63.

[16] Levine JD, Gordon NC, Jones RT, Fields HL. The narcotic antagonist naloxone enhances clinical pain. Nature 1978 Apr 27;272(5656):826-7.

[17] Lundeberg T, Bondesson L, Lundstrom V. Relief of primary dysmenorrhea by transcutaneous electrical nerve stimulation. Acta Obstet Gynecol Scand 1985;64(6):491-7.

[18] Marchand S, Li J, Charest J. Effects of caffeine on analgesia from transcutaneous electrical nerve stimulation. N Engl J Med 1995 Aug 3;333(5):325-6.

[19] Melzack R, Wall PD. Pain mechanisms: a new theory. Science 1965 Nov 19;150(699):971-9.

[20] O'Sullivan SB, Schmitz TJ. Physical Rehabilitation: Assessment and Treatment. Philadelphia: F. A. Davis Company; 2000.

[21] Robinson AJ, Snyder-Mackler L. Clinical Electrophysiology : Electrotherapy and Electrophysiologic Testing. 3rd ed. Philadelphia: Lippincott Williams \& Wilkins; 2007.

[22] Salar G, Job I, Mingrino S, Bosio A, Trabucchi M. Effect of transcutaneous electrotherapy on CSF beta-endorphin content in patients without pain problems. Pain 1981 Apr;10(2):169-72.

[23] Sjolund B, Terenius L, Eriksson M. Increased cerebrospinal fluid levels of endorphins after electro-acupuncture. Acta Physiol Scand 1977 Jul;100(3):382-4.

[24] Sjolund BH, Eriksson MB. The influence of naloxone on analgesia produced by peripheral conditioning stimulation. Brain Res 1979 Sep 14;173(2):295-301.

[25] Sluka KA, Deacon M, Stibal A, Strissel S, Terpstra A. Spinal blockade of opioid receptors prevents the analgesia produced by TENS in arthritic rats. J Pharmacol Exp Ther 1999 May;289(2):840-6. 
[26] Sluka KA, Judge MA, McColley MM, Reveiz PM, Taylor BM. Low frequency TENS is less effective than high frequency TENS at reducing inflammationinduced hyperalgesia in morphine-tolerant rats. Eur J Pain 2000;4(2):185-93.

[27] Tousignant-Laflamme Y, Page S, Goffaux P, Marchand S. An experimental model to measure excitatory and inhibitory pain mechanisms in humans. Brain Res 2008 Jul 9.

[28] Warke K, Al-Smadi J, Baxter D, Walsh DM, Lowe-Strong AS. Efficacy of transcutaneous electrical nerve stimulation (tens) for chronic low-back pain in a multiple sclerosis population: a randomized, placebo-controlled clinical trial. Clin J Pain 2006 Nov;22(9):812-9.

[29] Willer JC, Roby A, Boulu P, Boureau F. Comparative effects of electroacupuncture and transcutaneous nerve stimulation on the human blink reflex. Pain 1982 Nov;14(3):267-78. 